\title{
Who Will Guard the Guardians? Preventing Drug Diversion in Hospitals
}

\author{
Sumant Ranji, MD ${ }^{1 *}$
}

\begin{abstract}
${ }^{1}$ Division of Hospital Medicine, Zuckerberg San Francisco General Hospital, University of California San Francisco, San Francisco, California.
\end{abstract}
ran he patient safety field rightly focuses on identifying and addressing problems with systems of care. From the patient's perspective, however, underlying systems issues might be less critical than another unspoken question: can I trust the people who are taking care of me? Last year, a popular podcast ${ }^{1}$ detailed the shocking story of Dallas neurosurgeon Christopher Duntsch, who was responsible for the death of two patients and severe injuries in dozens of other patients over two years. Although fellow surgeons had raised concerns about his surgical skill and professionalism almost immediately after he entered practice, multiple hospitals allowed him to continue operating until the Texas Medical Board revoked his license. Duntsch was ultimately prosecuted, convicted, and sentenced to life imprisonment, in what is believed to be the first case of a physician receiving criminal punishment for malpractice.

Only a small proportion of clinicians repeatedly harm patients as Duntsch did, and the harm they cause accounts for only a small share of the preventable adverse events that patients experience. Understandably, cases of individual clinicians who directly harm patients tend to capture the public's attention, as they vividly illustrate how vulnerable patients are when they entrust their health to a clinician. As a result, these cases have a significant effect on the patient's trust in healthcare institutions.

In this issue of the Journal of Hospital Medicine ${ }^{\circledR}$, Fan and colleagues ${ }^{2}$ describe the problem of controlled-substance diversion in hospitals and review the contributors and potential solutions to this issue. Their thorough and insightful review highlights a growing problem that is probably invisible to most hospitalists. Diversion of controlled substances can happen at any stage of the medication use process, from procurement to disposal, and drugs can be diverted by healthcare workers, nonclinical staff, patients, and caregivers. Perhaps most concerning to hospitalists, diversion at the prescribing and administration stages can directly affect patient care. Strategies used to individualize pain control, such as using flexible dose ranges for opioids, can be manipulated to facilitate diversion at the expense of the patient's suffering.

The review presents a comprehensive summary of safeguards against diversion at each stage of the medication use process and appropriately emphasizes system-level solutions.

*Corresponding Author: Sumant Ranji, MD; E-mail: sumant.ranji@ucsf.edu; Telephone: 415-206-2651; Twitter @sumantranji

Published online first June 12, 2019.

Received: May 23, 2019; Accepted: May 24, 2019

๑) 2019 Society of Hospital Medicine DOI 10.12788/jhm.3252
These include analyzing electronic health record data to identify unusual patterns of controlled substance use and developing dedicated diversion investigation teams. These measures, if implemented, are likely to be effective at reducing the risk of diversion. However, given the complexity of medication use, eliminating this risk is unrealistic. Opioids are used in more than half of all nonsurgical hospital admissions; ${ }^{3}$ although this proportion may be decreasing due to efforts to curb opioid overprescribing, many hospitalized patients still require opioids or other controlled substances for symptom control. The opportunity to divert controlled substances will always be present.

Eliminating the problem of drug diversion in hospitals will require addressing the individuals who divert controlled substances and strengthening the medication safety system. The term "impaired clinician" is used to describe clinicians who cannot provide competent care due to illness, mental health, or a substance-use disorder. In an influential 2006 commentary, Leape and Fromson made the case that physician performance impairment is often a symptom of underlying disorders, ranging from short-term, reversible issues (eg, an episode of burnout or depression) to long-term problems that can lead to permanent consequences (eg, physical illness or substance-use disorders). ${ }^{4}$ In this framework, a clinician who diverts controlled substances represents a particularly extreme example of the broader problem of physicians who are unable to perform their professional responsibilities.

Leape and Fromson called for proactively identifying clinicians at risk of performance failure and intervening to remediate or discipline them before patients are harmed. To accomplish this, they envisioned a system with three key characteristics:

- Fairness: All physicians should be subject to regular assessment, and the same standards should be applied to all physicians in the same discipline.

- Objectivity: Performance assessment should be based on objective data.

- Responsiveness: Physicians with performance issues should be identified and given feedback promptly, and provided with opportunities for remediation and assistance when underlying conditions are affecting their performance.

Some progress has been made toward this goal, especially in identifying underlying factors that predispose to performance problems. ${ }^{5}$ There is also greater awareness of underlying factors that may predispose to more subtle performance deterioration. The recent focus on burnout and well-being among physicians is long overdue, and the recent Charter on Physician Well-Being ${ }^{6}$ articulates important principles for healthcare or- 
ganizations to address this epidemic. Substance-use disorder is a recognized risk factor for performance impairment. Physicians have a higher rate of prescription drug abuse and a similar overall rate of substance-use disorders compared to the general population. While there is limited research around the risk factors for drug diversion by physicians, qualitative studies ${ }^{7}$ of physicians undergoing treatment for substance-use disorders found that most began diverting drugs to manage physical pain, emotional or psychiatric distress, or acutely stressful situations. It is plausible that many burned out or depressed clinicians are turning to illicit substances to self-medicate increasing the risk of diversion.

However, 13 years after Leape and Fromson's commentary was published, it is difficult to conclude that their vision has been achieved. Objectivity in physician performance assessment is still lacking, and most practicing physicians do not receive any form of regular assessment. This places the onus on members of the healthcare team to identify poorly performing colleagues before patients are harmed. Although nearly all states mandate that physicians report impaired colleagues to either the state medical board or a physician rehabilitation program, healthcare professionals are often reluctant ${ }^{8}$ to report colleagues with performance issues, and clinicians are also unlikely ${ }^{9}$ to self-report mental health or substance-use issues due to stigma and fear that their ability to practice may be at risk.

Even when colleagues do raise alarms - as was the case with Dr. Duntsch, who required treatment for a substance-use disorder during residency-existing regulatory mechanisms either lack evidence of effectiveness or are not applied consistently. State licensing boards play a crucial role in identifying problems with clinicians and have the power to authorize remediation or disciplinary measures. However, individual states vary widely ${ }^{10}$ in their likelihood of disciplining physicians for similar offenses. The board certification process is intended to ensure that only fully competent physicians can practice medicine independently. However, there is little evidence that the certification process ensures that clinicians maintain their skills, and significant controversy has accompanied efforts to revise the maintenance of certification process. The medical malpractice system aims to improve patient safety by ensuring compensation when patients are injured and by deterring substandard clinicians from practicing. Unfortunately, the system often fails to meet this goal, as malpractice claims are rarely filed even when patients are harmed due to negligent care. ${ }^{11}$

Given the widespread availability of controlled substances in hospitals, comprehensive solutions must incorporate the systems-based solutions proffered by Fan and colleagues and address individual clinicians (and staff) who divert drugs. These clinicians are likely to share some of the same risk factors as clinicians who cannot perform their professional responsibilities for other reasons. Major system changes are necessary to minimize the risk of short-term conditions that could affect physician performance (such as burnout) and develop robust methods to identify clinicians with longer-term issues affecting their performance (such as substance-use disorders).

Although individual clinician performance problems likely account for a small proportion of adverse events, these issues strike at the heart of the physician-patient relationship and have a profound impact on patients' trust in the healthcare system. Healthcare organizations must maintain transparent and effective processes for addressing performance failures such as drug diversion by clinicians, even if these processes are rarely deployed.

Disclosures: The author does not have any conflict of interest to report.

\section{References}

1. "Dr. Death" (podcast). https://wondery.com/shows/dr-death/. Accessed May 16, 2019

2. Fan $M$, Tscheng D, Hyland $B$, et al. Diversion of controlled drugs in hospitals: a scoping review of contributors and safeguards. J Hosp Med. 2019;14(7):419428. doi: 10.12788/jhm.3228

3. Herzig SJ, Rothberg MB, Cheung M, Ngo LH, Marcantonio ER. Opioid utilization and opioid-related adverse events in nonsurgical patients in US hospitals. J Hosp Med. 2014;9(2):73-81. doi: 10.1002/jhm.2102.

4. Leape LL, Fromson JA. Problem doctors: is there a system-level solution? Ann Intern Med. 2006;144(2):107-115. doi: 10.7326/0003-4819-144-2200601170-00008.

5. Studdert DM, Bismark MM, Mello MM, et al. Prevalence and characteristics of physicians prone to malpractice claims. N Engl J Med. 2016;374(4):354362. doi: 10.1056/nejmsa1506137.

6. Thomas LR, Ripp JA, West CP. Charter on physician well-being. JAMA. 2018;319(15):1541-1542. doi: 10.1001/jama.2018.1331.

7. Merlo LJ, Singhakant S, Cummings SM, Cottler LB. Reasons for misuse of prescription medication among physicians undergoing monitoring by a physician health program. J Addict Med. 2013;7(5):349-353. doi: 10.1097/ adm.0b013e31829da074.

8. DesRoches CM, Fromson JA, Rao SR, et al. Physicians' perceptions, preparedness for reporting, and experiences related to impaired and incompetent colleagues. JAMA. 2010;304(2):187-193. doi: 10.1001/jama.2010.921.

9. Samuel L. Doctors fear mental health disclosure could jeopardize their licenses. STAT. October 16, 2017. https://www.statnews.com/2017/10/16/doctors-mental-health-licenses/. Accessed May 16, 2019.

10. Harris JA, Byhoff E. Variations by the state in physician disciplinary actions by US medical licensure boards. BMJ Qual Saf. 2017;26(3):200-208. doi:10.1136/ bmjqs-2015-004974.

11. Studdert DM, Thomas EJ, Burstin HR, et al. Negligent care and malpractice claiming behavior in Utah and Colorado. Med Care. 2000;38(3):250-260. doi:10.1097/00005650-200003000-00002 